\title{
Attitudes to mouthguards and prevalence of orofacial injuries in four teams competing at the second Rugby World Cup
}

\author{
P. J. Chapman MBBS MDS FASMF and B. P. Nasser* BDSc \\ Department of Oral and Maxillofacial Surgery, University of Queensland Dental School, Brisbane, Australia
}

\begin{abstract}
The opportunity was taken to conduct a questionnaire survey of the members of four competing countries at the second Rugby World Cup tournament (Australia, Ireland, Scotland, Wales) regarding the prevalence of orofacial injuries and attitudes to mouthguards. The results show that while all players in each team believed that mouthguards provided local protection, approximately one in five of each team did not wear one. The average age when players first started wearing mouthguards varied from 12.7 years for Australia to 18.1 years for Wales, while the percentage of wearers willing to play without their mouthguard ranged from only $4.6 \%$ for Australia to $15.8 \%$ for Wales. Just less than half of the total group had previously sustained an orofacial injury playing rugby.
\end{abstract}

Keywords: Rugby World Cup, orofacial injuries, mouthguard usage

It is generally accepted that the risk of sustaining orofacial injures in rugby is greatest in the higher grades and international rugby ${ }^{1,2}$. Orofacial injuries include fractures of the jaws and orodental injuries, the latter term including dental and dentoalveolar injuries and intraoral and perioral lacerations. A professionally fitted mouthguard significantly reduces the risk of sustaining orodental injuries ${ }^{3,4}$. Additionally the mouthguard will reduce the incidence of impact acceleration head injuries (concussion) following impact to the master mandible from below $^{5}$. This is relevant to preventive sports medicine because the most common cause of concussion in sports is a blow to the mandible ${ }^{5}$. Because mouthguards provide safety for participants in rugby, Tomasin et al. ${ }^{6}$ recently stated that 'Although little equipment is used in rugby, all players should wear a protective mouthpiece'.

Over-the-counter and professionally fitted mouthguards have a similar design and are made of similar material but provide different levels of protection. A professionally fitted mouthguard which is formed on

Address for correspondence: Dr P. J. Chapman, Department of Oral and Maxillofacial Surgery, University of Queensland Dental School, Brisbane, Australia

(C) 1993 Butterworth-Heinemann Ltd 0306-3674/93/030197-03 a model of the athlete's upper jaw and fitted by a dentist is far superior in all aspects to the over-thecounter types. It has good retention, adequate maxillary bone coverage which increases energy dissipation, and a high user acceptance rate. An over-the-counter mouthguard, either self-moulded ('boil and bite') or pre-moulded ('stock'), may have insufficient retention to prevent dislodgement or displacement during impact, which would render it ineffective when protection was needed ${ }^{4}$. This inadequacy regarding retention especially applies to the pre-moulded type which requires the teeth to be clenched to be held in position ${ }^{7}$. Further, if dislodged the mouthguard could cause obstruction of the upper airway, especially if the athlete was concussed in the incident $^{3,7}$. An over-the-counter mouthguard also has inadequate coverage of the maxillary bone. Finally, it has a low user acceptance rate, particularly the pre-moulded type and most wearers do not continue wearing it for long. Although the professionally fitted mouthguard costs considerably more, the cost-benefit ratio clearly shows it to be a worthwhile investment and numerous studies have shown that it reduces the incidence, severity and extent of orodental injuries ${ }^{1,2,7-9}$. Therefore, health professionals should generally recommend use of professionally fitted mouthguards for athletes involved in contact sports. Protection for young teenagers and children where mouthguards need replacement every 1 or 2 years can alternatively be provided by having a dentist or suitably trained person correctly fit a self-moulded type of mouthguard $^{3}$. It should be recalled that the risk of injury and levels of impact and severity and extent of injury are much less in this group compared with adolescent and adult sport, and this option is safer and preferable to not wearing a mouthguard 4 . Finally a pre-moulded mouthguard should never be recommended.

The aim of this study was to contribute to ongoing research into mouthguard usage patterns and orofacial injury in international rugby.

\section{Methods}

A pre-tested questionnaire was distributed to the members of four of the teams competing in the 
second Rugby World Cup (Australia, Scotland, Ireland, Wales) by the second author, a member of the Australian squad. The questionnaire sought information about players' attitudes to wearing mouthguards, as well as details of any orofacial injuries they had previously sustained when playing rugby which had required treatment, either medical or dental.

\section{Results}

The results of the questionnaire are presented in Table 1. Of the orodental injuries sustained, $22.2 \%$ were soft tissue lacerations and $77.8 \%$ were dental injuries. Of the dental injuries, $84.8 \%$ involved maxillary incisor teeth, $9.1 \%$ involved mandibular incisor teeth and $6.1 \%$ involved posterior teeth. Treatment varied from minor restorative procedures to extensive endodontics and occasional extractions. The majority of orodental injuries were sustained by forwards $(59.2 \%)$ compared with backs while there were three instances of a fractured mandible which interestingly were all sustained by backs, i.e. two in the Australian team (a halfback and a winger) and one in the Irish team (a winger).

\section{Discussion}

The results show that there was little or no difference between the teams regarding the following aspects of the questionnaire: average age of players; average age when first started playing rugby; average number of years each player had played rugby; percentage who believe mouthguards provide local protection; percentage who wore a mouthguard; percentage of mouthguards which were professionally fitted.

The responses which showed greatest variations between individual teams were: average age when players started wearing mouthguards ranged from 12.7 years for Australia to 18.1 years for Wales; average delay from starting to play rugby before each wearer started wearing a mouthguard ranged from 3.2 years for Australia to 7.2 years for Scotland; percentage of mouthguard wearers who would not play without it ranged from $27.3 \%$ for Australia to $54.6 \%$ for Ireland; percentage of mouthguard wearers willing to play without it ranged from $4.6 \%$ for Australia to $15.8 \%$ for Wales; percentage of wearers who believe mouthguards should be compulsory for adult rugby players ranged from $45.5 \%$ for Scotland to $86.4 \%$ for Ireland; percentage of players who had previously sustained an orodental injury ranged from $26.9 \%$ for Ireland to $54.2 \%$ for Wales; the percentage of such players who were wearing a mouthguard at the time ranged from none for Wales to $36.4 \%$ for Australia; the percentage of players who had sustained a fractured mandible ranged from none for Scotland and Wales to $7.7 \%$ for Australia.

Although all members of the four teams believed that mouthguards provided local protection, approximately one in five in each team did not wear one. Of the 16 players who did not currently wear a mouthguard, six had never worn one; six had tried and discarded an over-the-counter type because of the difficulty when wearing it; and four had previously tried a professionally fitted mouthguard and later stopped wearing it - their complaints were uncomfortable fit, feeling of nausea, and difficulty with speaking and breathing - these problems could have been corrected by the mouthguard being adjusted by a dentist, or replaced, and the players getting used to wearing it at training sessions. It would be rare that a player could not be satisfactorily fitted with a professionally fitted mouthguard. Further, players should be encouraged to see their dentist if they are having any problems when wearing their mouthguard.

Although the incidence of orodental injuries sustained when wearing a mouthguard was as high as $36.4 \%$ in one team, it should be stressed that the extent and severity of these injuries would have been considerably greater if a mouthguard had not been

Table 1. Summary of questionnaire results

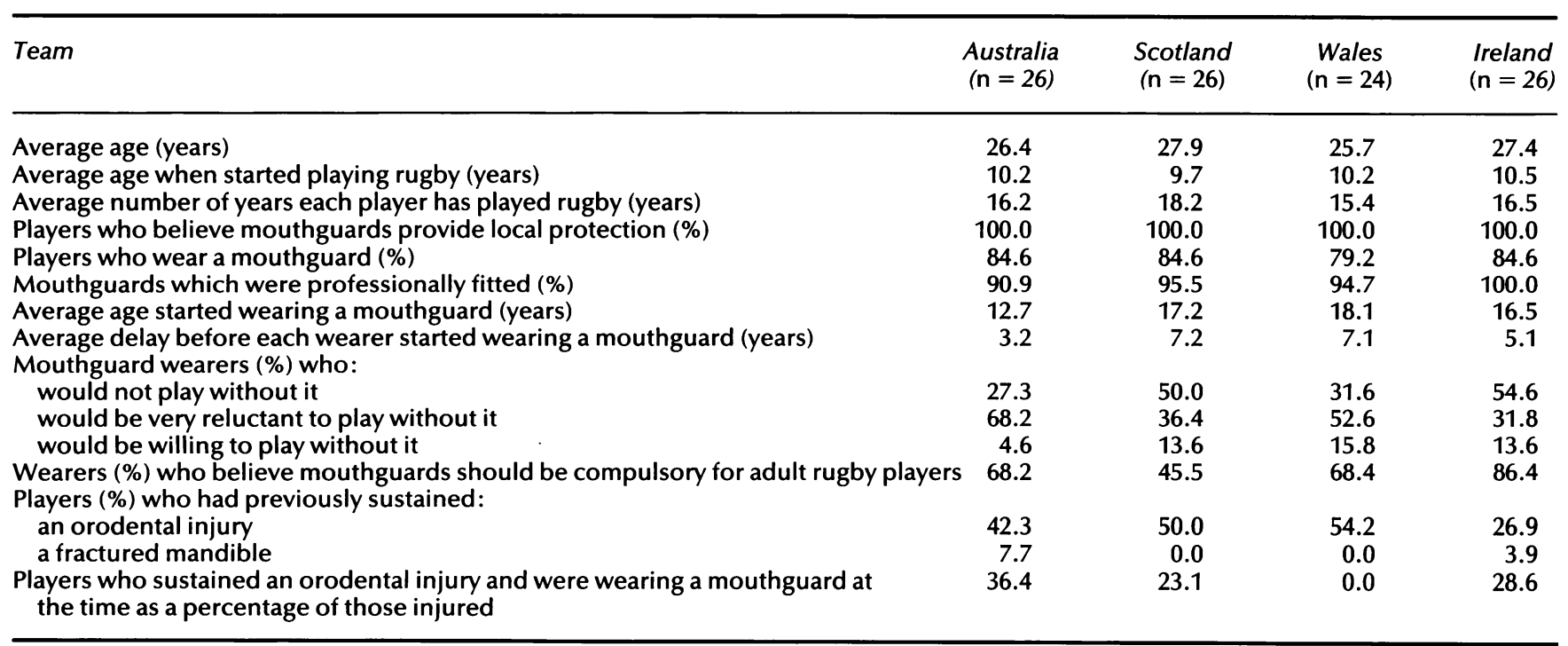


worn. By contrast, none of those injured in another team were wearing a mouthguard at the time. Also, the findings show a reversal of the usual pattern of occurrence of fractures of the mandible with all being sustained by backs.

A recent innovation, the professionally fitted bimaxillary mouthguard, provides increased orodental protection as well as protection against sustaining a fractured mandible $e^{10}$. Ten of the Australian team wore one, nine of whom were forwards, for an average of 3.2 years (range 1-6 years) and no instances of injuries requiring treatment have occurred. It is mainly suggested as an option in higher grades of competition and for athletes returning to competition following a fracture of the mandible. None of the wearers would revert to a standard mouthguard because of the increased protection they feel it provides.

\section{Acknowledgements}

The authors greatly appreciate the willing cooperation of the players involved in this study.

\section{References}

1 Chapman PJ. Orofacial injuries and mouthguards: a study of the 1984 Wallabies. Br J Sports Med 1984; 19: 93-5.

2 Chapman PJ. The prevalence of orofacial injuries and use of mouthguards in rugby union. Aust Dent $J 1985 ; 30$ : 364-7.

3 Chapman PJ. Mouthguards and the role of sporting team dentists. Aust Dent J 1989; 34: 36-43.

4 Chapman PJ. Prevention of orofacial sporting injuries. Dentistry Today 1990; 6: 1-8.

5 Chapman PJ. Concussion in contact sports and importance of mouthguards in protection. Aust J Sci Med Sport 1985; 17: 23-7.

6 Tomasin JD, Martin DF, Curl WW. Recognition and prevention of rugby injuries. Phys Sports Med 1989; 17: 114-26.

7 Chapman PJ. Attitudes to mouthguards and prevalence of orofacial injuries in international rugby: a study of the 1990 Wallabies. Aust J Sci Med Sport 1991; 23: 113-5.

8 Kay EJ, Kakarla P, Macleod DAD, McGlashan TPL. Orofacial and dental injuries in club rugby union players. $\mathrm{Br} J$ Sports Med 1990; 24: 271-3.

9 Jennings DC. Injuries sustained by users and non-users of gum shields in local rugby union. Br J Sports Med 1990; 24: 159-65.

10 Chapman PJ. Fabricating a bimaxillary mouthguard. Aust Dent Assoc Newsletter (Vic ed) 1992; 10: 20-1. 